\title{
A SMART, LOCATION BASED TIME AND ATTENDANCE TRACKINg SYSTEM USING ANDROID APPLICATION
}

\author{
Shermin Sultana ${ }^{1}$, Asma Enayet ${ }^{1}$ and Ishrat Jahan Mouri ${ }^{1}$ \\ ${ }^{1}$ Department of Computer Science and Engineering, Stamford University Bangladesh, \\ Dhaka, Bangladesh
}

\begin{abstract}
Over the years the process of manual attendance has been carried out which is not only time consuming but also provides erroneous result. Automated time and attendance monitoring system provides many benefits to organizations. This reduces the need of pen and paper based manual attendance tracking system. Following this thought, we have proposed a smart location based time and attendance tracking system which is implemented on android mobile application on smartphone reducing the need of additional biometric scanner device. The location of an organization has a specific location, which can be determine by the GPS. Each employee's location can be determined by the GPS using smartphone. This location is defined as a key of time and attendance tracking in our paper.
\end{abstract}

\section{KEYWORDS}

Location-based service, GPS, time and attendance system, sending SMS, android applications.

\section{INTRODUCTION}

Now a day, attendance monitoring and working hour calculation is very essential for almost every institution or organization. Typically there are two types of attendance system available, i) Manual and ii) Automated. Manual system involves the use of sheets of paper or books in taking attendance where employees fill out and managers oversee for accuracy. This method could be erroneous because sheets could be lost or damaged. Also the extraction of relevant data and the manual computation of working time is very time consuming. It takes an extra employee to check for the attendance and timing of other employees which includes cost overhead for the organization as well [1].

On the other hand, automated time and attendance systems implies the use of electronic tags, barcode badges, magnetic stripe cards, biometrics (hand, fingerprint, or facial), and touch screens [2] in place of paper sheets. In these aforementioned techniques, employees touch or swipe in order to provide their identification and also the entering and leaving time to calculate working hours. The provided information are recorded and automatically transferred to a computer for processing. Using an automated system for time and attendance monitoring reduces the errors of manual system and conserve optimal amount of time. But these automated systems require heterogeneous devices need to be located in the organization which is costly.

In this paper, considering the wide popularity of smartphones, we introduce the use of smartphone for this time and attendance tracking purpose. We have proposed a location based smart time and attendance tracking system based on the concept of web services which is

DOI : 10.5121/ijcseit.2015.5101 
implemented as an Android mobile application that communicates with the remote server in which the database is located. Internet connectivity (Wi-Fi/3G) is needed for connecting to database residing in the remote server. Our proposed smart system does not require any kind of peripheral device other than smartphone which will reduce computational time and cost of placing an extra device. Any employee crossing the area border with a smartphone and the running application will be tracked automatically.

\section{RELATED WORKS}

Several techniques and methods have been carried out effectively to monitor employee attendance. Lawson et al. [3] proposed a cost effective computer based embedded attendance management system by which authority electrically monitors the attendance for verification using an improvised electronic card. These cards contain necessary information of an individual. These are inserted in an electronic machine which will record the time and other information to a server system. Password based authentication and verification of attendance monitoring system of any individuals has also been carried out in the literature. A system that applies user id and password of a person for authentication was designed and implemented by Cheng et al. [4].

However, an issue with these electronic cards or password based system allows for imposture since cards or passwords can be shared or someone can ask other person to insert his/her card or password. This problem can be addressed by using biometric recognition system which includes finger print or iris recognition.

A system was proposed and implemented by authors in [5] fingerprints to identify and calculate the attendance and generate the reports after a fixed time duration. Individuals simply put their fingerprints on the fingerprint reader which scans the finger print and verifies that person. M. Smaili et al [6] solved the problem by proposing a wireless attendance management system where iris of an individual is used for authentication. It is also like fingerprint where no two people can have the same eyes. A scanner will scan the eyes and automatically log the person in. Unlike fingerprint, iris is more preserved from the external environment. But both the fingerprint and iris recognition based approach needs some extra devices or scanner which can be connected to the server computation system.

In our work, we addressed the problem utilizing smartphones internet connectivity for monitoring the presence or attendance of an individual. Smartphone based monitoring system reduces the surplus cost of additional scanning device because now a days almost each employee possess a smartphone of his own. An area is fixed for every employee when an employee enters or exits that area, that time stamp is saved and the time duration of any particular employee residing within its area is calculated by the system.

\section{Proposed ATtendance Tracking System Overview ANd Design}

\subsection{System Overview:}

The proposed system provides a solution to manual attendance taking problem. This system is a location based smart time and attendance tracking system based on the concept of web services which is implemented as an android mobile application. The employee has to install the respective APK files developed for them on their android devices. At first it is important to save the office coordinates by entering the latitude, longitude and radius of area. Employee has to save the IP (internet protocol) address of the office internet. At the same time one employee can save their information through the info menus of the App. 
This location based time and attendance tracking system locates your position and logs your login and logout time. As the employee enters his workplace area, the system connects to the office internet and sends the employee id and local time to the server. Then the server gets the local time and stores the information in a database. Again when employee leaves the office area, the system notifies the office server that the employee is leaving. To run the whole system accurately it is important that both the employee device and office server is in the same internet connection (Wi$\mathrm{Fi} / 3 \mathrm{G})$.

\subsection{System Design:}

The smart, location based time and attendance tracking system is a client-server approach and follows specific hardware and software architecture. Integrating the hardware and software is the main challenge here and the hardware and software works together.

The whole system has been divided into two major categories 1) App for mobile 2) App for pc

\subsubsection{Software Architecture}

The software architecture consists of: the database, the application program and the server.

- Database: The database consists of a number of tables, which stores records. We used apache derby database which is easy, fast and efficient and can store a large number of records and requires a little configuration.

- Application Program: The application program is developed with Android programming language using Eclipse framework. The application program provides user interface to both the employees and office server. Programming in Android is simple, user friendly and android offers an excellent data connectivity.

- Server: The server is deployed on the personal computer using apache-Tomcat7. Tomcat7 is free, robust and easy to deploy.

\subsubsection{Hardware Architecture}

The basic requirement of the location base time and attendance tracking system is an android device, which will run the application, with the help of which the employees will mark their attendance and take their login, logout time automatically without any hassle. The other requirement is a personal computer on the server side, which will store the database.

\section{METHODOLOGY}

User authentication is one of the major factors in the proposed system. Every employee is authenticated based on his/her unique user identification number. This unique identification number is the number which is given by the office. The identification number along with other information is also saved in the employee device.

At first employee has to install the required system APK files into their android device. Mobile location service has to be on when the system was running. If mobile location service is off then the whole process will not go further. Mobile location service helps to trace the employee location. When the employee enters the office area, android device of the employee is automatically connected to the office internet and a message is sent to the office sever with the employee id and local time of the device which is counted as login time of that employee. When employee leaves the office area, a message is sent to the office server with employee id and local time which is counted as logout time. Figure 1 depicts the overall methodology of our proposed system. 


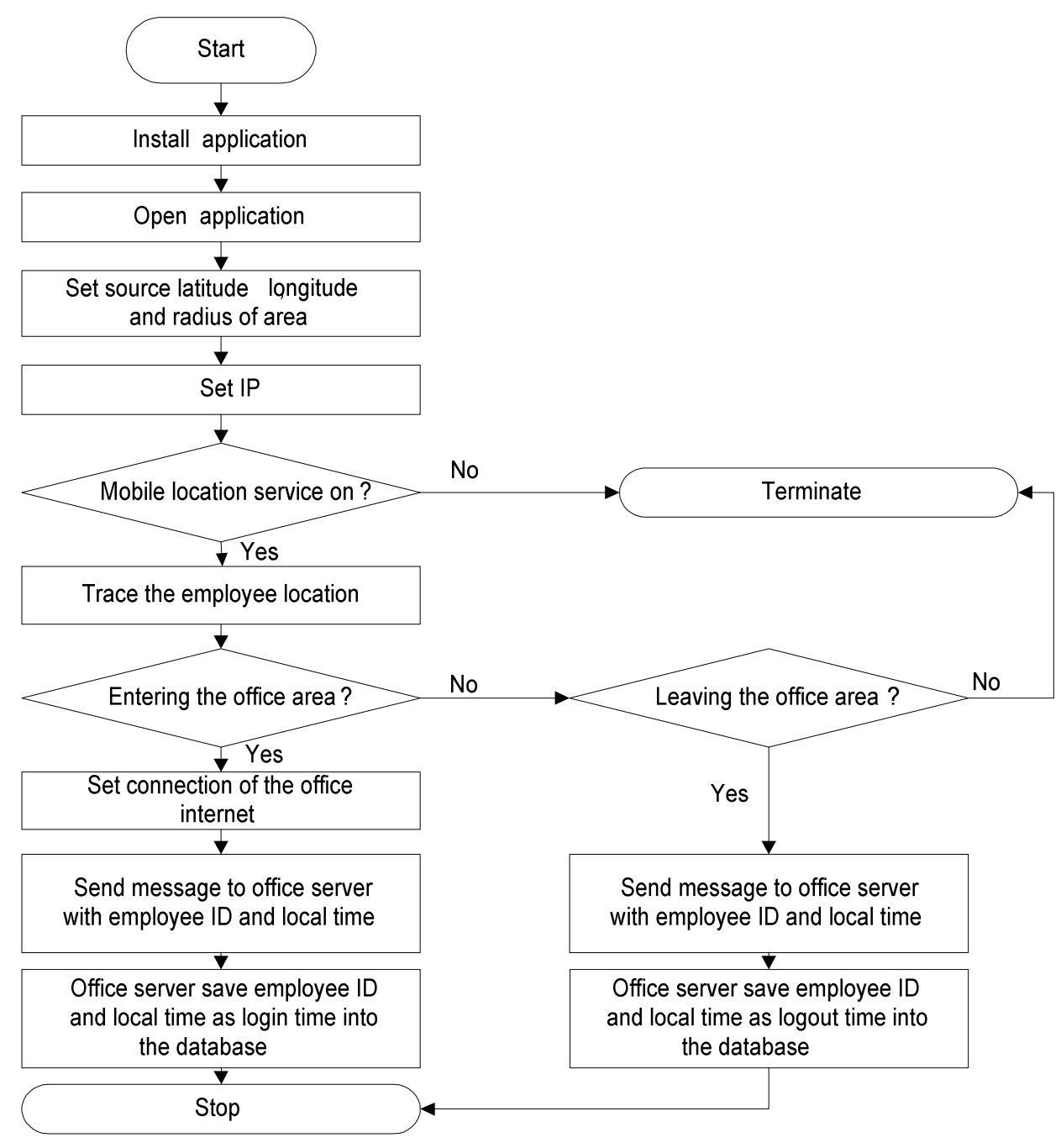

Figure 1. Flow of operation for the proposed system

\section{CONCLUSION}

This paper introduce a smart, location based time and attendance tracking system using android application which use location as the core component of attendance tracking using smartphone. The area is set for tracking using GPS and employee coordinate inside the area border depicts that employee is present in the organization. We developed this system for android platform, but we are focusing on developing this system for iOS platform as well in neat future.

\section{ACKNOWLEDGEMENTS}

The authors would like to thank everyone, just everyone! 


\section{REFERENCES}

[1] Kumbhar, A. A., Wanjara, K. S., Trivedi, D. H., Khairatkar, A. U., \& Sharma, D. (2014). “Automated Attendance Monitoring System using Android Platform.”, International Journal of Current Engineering and Technology, Vol. 4, No. 2, pp1096-1099.

[2] Pankanti, S., Prabhakar, S., \& Jain, A. K. (2002). "On the individuality of fingerprints", Pattern Analysis and Machine Intelligence, IEEE Transactions on, 24(8), 1010-1025.

[3] Shoewu, O. O. M. Olaniyi, and Lawson (2011), "Embedded Computer-Based Lecture Attendance Management System”, African Journal of Computing and ICT (Journal of IEEE Nigeria Computer Section), 4(3):27 - 36.

[4] Cheng, K., L. Xiang, T. Hirota, and K. Ushijimaa (2005), "Effective Teaching for Large Classes with Rental PCs by Web System WTS”, Pro. Data Engineering Workshop (DEWS2005), 1D - d3 (in Japanese).

[5] Shoewu, O. and O.A. Idowu (2012), "Development of Attendance Management System using Biometrics", Pacific Journal of Science and Technology, 13(1):300-307.

[6] Kadry, S., \& Smaili, M. (2013). "Wireless attendance management system based on iris recognition", Scientific Research and essays, 5(12), 1428-1435.

[7] Android Developer Guide: http://developer.android.com/guide/index.html accessed at 18th January.

[8] Android API: http://developer.android.com/reference/packages.html accessed at 20th January.

[9] Android Developers blog: http://android-developers.blogspot.com/ accessed at 15th January.

\section{Authors}

Asma Enayet is a lecturer in the Department of Computer Science and Engineering, Stamford University Bangladesh, Dhaka, Bangladesh. She received her B.Sc in Computer Science and Engineering from University of Dhaka, Bangladesh in 2012. She passed her Higher Secondary Certificate (H.S.C) and Secondary School Certificate (S.S.C) form Holy Cross College, Dhaka, Bangladesh in 2008 and Holy Cross Girls' High School, Dhaka, Bangladesh in 2006. She published several journal and international conference paper from her research work. Her research interest includes web service composition, semantic web service, knowledge representation, mobile apps, etc.

Shermin Sultana is a lecturer in the Department of Computer Science and Engineering, Stamford University Bangladesh, Dhaka, Bangladesh. She received her B.Sc in Computer Science and Engineering from Ahsanullah University of Science and Technology, Dhaka, Bangladesh in 2012. She passed her Higher Secondary Certificate (H.S.C) and Secondary School Certificate (S.S.C) from Ideal School and College, Motijheel, Dhaka, Bangladesh in 2008 and Kamrunnesa Govt Girls High School, Dhaka, Bangladesh in 2006. Her research interest includes Artificial Intelligence, Mobile Apps and Neural Network etc.

Israt Jahan Mouri is a lecturer in the Department of Computer Science and Engineering, Stamford University Bangladesh, Dhaka, Bangladesh. She received her B.Sc in Computer Science and Engineering from Military Institute of Science \& Technology (MIST), Dhaka, Bangladesh in 2014. She passed her Higher Secondary Certificate (H.S.C) and Secondary School Certificate (S.S.C) from Rajuk Uttara Model College, Dhaka, Bangladesh in 2009 and The Buds Residential Model College, Sylhet, Bangladesh in 2007. Her research interest includes Pattern Recognition, Mobile Apps and Neural Network etc.
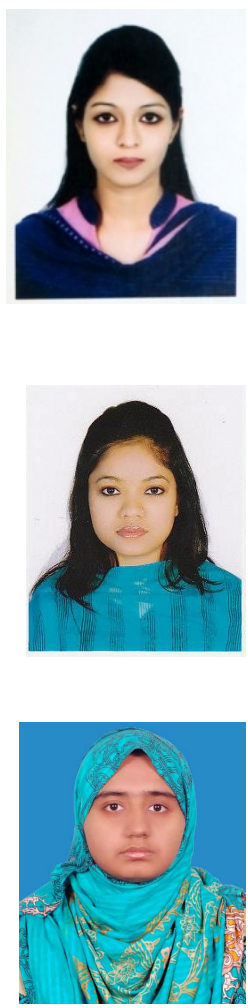